Please do not remove this page

RMIT

UNIVERSITY

\title{
Accurate control of stoichiometry and doping in barium stannate perovskite oxide nanoparticles
}

Shepherd, William; Wilms, Michael; van Embden, Joel; Della Gaspera, Enrico

https://researchrepository.rmit.edu.au/esploro/outputs/9921863703501341/filesAndLinks?institution=61RMIT_INST\&index=null

Shepherd, W., Wilms, M., van Embden, J., \& Della Gaspera, E. (2019). Accurate control of stoichiometry and doping in barium stannate perovskite oxide nanoparticles. Chemical Communications, 55(79), 11880-11883. https://doi.org/10.1039/C9CC04838C Document Version: Accepted Manuscript

Published Version: https://doi.org/10.1039/C9CC04838C 


\section{Stoichiometry and Doping in}

\section{Accurate Control of}

Barium stannate $\left(\mathrm{BaSnO}_{3}\right)$ is one of the most promising emerging materials for use as a transparent electrode. However, to date, its synthesis has been proven to be highly irreproducible. In this communication, we present a detailed investigation of the reproducibility issues and provide a robust approach to synthesize $\mathrm{BaSnO}_{3}$ nanomaterials with controlled stoichiometry and doping.

Barium stannate $\left(\mathrm{BaSnO}_{3}, \mathrm{BSO}\right)$ is a perovskite oxide that has recently emerged as an alternative to indium tin oxide (ITO) for use in optoelectronic devices. ${ }^{1}$ Its large band gap in the near UV ( 3.1 eV) guarantees optical transparency at visible and near infrared (NIR) wavelengths, while its exceptional electron mobility (experimentally demonstrated up to $320 \mathrm{~cm}^{2} \mathrm{~V}^{-1} \mathrm{~s}^{-1}$ ) makes it appealing for enhanced electrical conductivity, especially when BSO is doped with aliovalent cations, such as lanthanum. ${ }^{2,3,4,5}$ Despite such recent interest, the methods currently available for the synthesis of BSO are limited and rely on vacuum depositions (molecular beam epitaxy, sputtering), or conventional ceramic processes (solid state reactions at high temperatures), which are restricted in the ability to tune the properties of the prepared products. ${ }^{6,7,8,9}$ On the other hand, wet chemistry methods can provide great control on the chemical, morphological and functional properties of the final material. Currently available solution-based approaches are mostly based on the precipitation of a mixed Ba-Sn precursor through the addition of ammonia to a solution of barium and tin salts, followed by high temperature annealing. ${ }^{10,11,12,13,14} \mathrm{~A}$ recent study by Shin et al. detailed the formation of a Crystalline Superoxide Molecular Cluster (CSMC) precursor, a crystalline solid with a simple cubic structure which converts into $\mathrm{BaSnO}_{3}$ at low temperatures $\left(200-300^{\circ} \mathrm{C}\right) .{ }^{15}$ Although this work built on previous studies which outlined similar syntheses and low conversion temperatures, 10,14 it was the first to identify the nature of the precursor and to provide a pathway for the fabrication of BSO and lanthanum-doped BSO (LBSO) at far lower temperatures compared to those required by conventional solid-state or co-precipitation reactions. $6,7,9,12,16$ Here, we present a detailed investigation into the CSMCmediated synthesis of $\mathrm{BaSnO}_{3}$ nanoparticles, and address several irreproducibility issues that have not been observed thus far. By solving these challenges, we are able to outline a robust, reproducible method to synthesize BSO nanoparticles with controllable stoichiometry and tuneable doping levels. First, we synthesized BSO using the CSMC-mediated route (see ESI for experimental details). ${ }^{15}$ Briefly, barium and tin chlorides are dissolved in aqueous hydrogen peroxide in the presence of citric acid and placed in a water bath at $60{ }^{\circ} \mathrm{C}$. The CSMC precursor is then precipitated with ammonium hydroxide solution and isolated by centrifugation. The successful synthesis of the CSMC precursor and of pure BSO after annealing was confirmed by $x$-ray diffraction (XRD) and Fourier transform infrared (FTIR) spectroscopy as shown in Figure 1. The shift in the diffraction peaks in the annealed material (Figure 1a) is due to the conversion of the peroxo (-OOH) and superoxo (-O-O-) species of the precursor into the cubic perovskite structure of BSO. ${ }^{15}$ Similarly, there is an evident decrease in the FTIR peaks associated with water and peroxo groups in the annealed samples compared to the CSMC (Figure $1 \mathrm{~b}$ ). The intense peaks at $630-700 \mathrm{~cm}^{-1}$ and $\sim 1430 \mathrm{~cm}^{-1}$ which pertain to $\mathrm{Sn}-\mathrm{O}$ and $\mathrm{Ba}-$ $O$ vibrations respectively, are readily observed in the annealed samples and provide additional evidence to the synthesis of BSO. ${ }^{10,17,18} \mathrm{~A}$ list of the vibrational peaks and their assignment is reported in the ESI as Table S1. The transformation of CSMC precursor into BSO is accompanied by a weight loss due to the removal of water, and the decomposition of peroxo/superoxo groups and of the organic ligand (citric acid) as confirmed by thermal gravimetric analysis (TGA, Figure S1).

Having confirmed the successful synthesis of pure phase BSO, we investigated the key aspects of this reaction, starting with the role of ammonia. Many of the available recipes do not specify the exact amount of ammonia, but broadly quote a suitable $\mathrm{pH}$ range. We have shown that the amount of ammonia is related to the crystallinity of $\mathrm{BSO}$, with a molar ratio $\mathrm{NH}_{3}:(\mathrm{Ba}+\mathrm{Sn}) \sim 40$ being optimal to achieve good crystallization (Figure S2). Notably, the quality of BSO was unaffected by the nature of the ammonia addition (rapid injection or dropwise addition, see Figure S3). For consistency, all results reported herein are achieved with the rapid injection of ammonia.
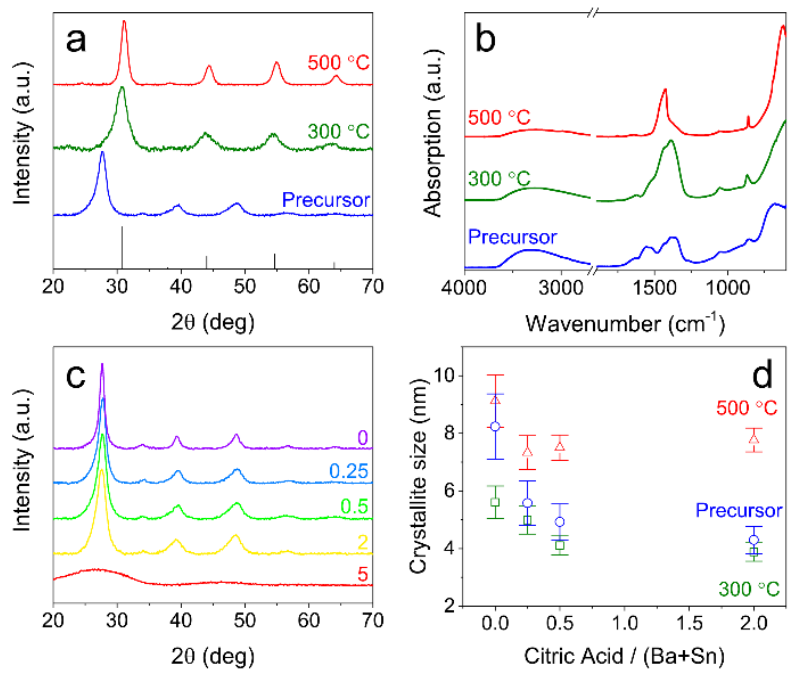

Figure 1. XRD patterns (a) and FTIR spectra (b) of the CSMC precursor and of BSO powders annealed at $300{ }^{\circ} \mathrm{C}$ and $500{ }^{\circ} \mathrm{C}$. The expected diffraction peak position for pure BSO (ICDD No. 15-0780) are reported at the bottom of panel (a). c) XRD patterns of CSMC precursor powders synthesized with varying amounts of citric acid. The reported values identify the citric acid / $(\mathrm{Ba}+\mathrm{Sn})$ molar ratio. d) Crystallite size of BSO precursor and annealed powders as a function of the amount of citric acid.

Another key aspect is the role of the ligands on the crystallinity of the CSMC. Most of the reported solution-based syntheses employ citric acid as ligand, without mentioning the effect of its concentration. By systematically varying the concentration of 
citric acid we found that it acts as a chelating

agent that limits, and eventually prevents the crystallization of the CSMC. As shown in Figure 1c, syntheses conducted with excess of citric acid (5:1 molar ratio with metals) produce amorphous powders. Decreasing the amount of citric acid is conducive to larger, more crystalline materials as evidenced by the sharpening of the diffraction peaks. This trend is also maintained in the annealed BSO. However, it is reduced and eventually counterbalanced at high temperatures $\left(500^{\circ} \mathrm{C}\right)$ by thermally-induced grain growth. This is summarised in Figure $1 d$, which shows the crystallite size as a function of the citric acid:metal ratio at different annealing temperatures (see Figure S4 for the corresponding XRD patterns). The nanocrystalline nature of the synthesized BSO powders can also be seen in the SEM images (Figure S5).

As mentioned earlier, we noted irreproducibility issues with this synthesis, specifically related to the Ba:Sn ratio. To uncover the source of this batch-to-batch variation we firstly mixed the precursors for the two metals in a stoichiometric $1: 1$ ratio (Ba:Sn), and over many repeated syntheses we observed excess $\mathrm{Sn}$ in our product. After careful investigation we found that this was directly linked to the time the metal precursors were left to stir in the hydrogen peroxide solution in the water bath, before the addition of ammonia. From now on we refer to this time as "standing time". As shown in Figure 2a, for standing times up to $15 \mathrm{~min}$ the $\mathrm{Sn}:$ Ba ratio in the purified powder is exactly $1: 1$, as expected from the initial reaction stoichiometry. However, for longer standing times the powders are tin-rich. This effect becomes more pronounced the longer the precursor solution was allowed to stand prior to ammonia addition. After 120 minutes of standing time, the ratio of Sn:Ba approached $~ 3: 1$. This is verified by elemental analysis of the supernatant after centrifugation and removal of the insoluble CSMC (Figure 2b). Larger excess of Ba was found in the supernatant with increased standing time, consistent with higher Sn incorporation in the precipitate. We initially ascribed this behavior to the role of citric acid in selectively complexing tin over barium. However, reactions without citric acid showed an even greater effect, with preferential tin precipitation occurring only a few minutes after preparing the precursor solutions (Figure $2 \mathrm{c}$ ). It has been shown that small variations of Sn amount in BSO dramatically affects its electrical conductivity. ${ }^{19}$ As such, it is critical to understand the underlying conditions that control stoichiometry in CSMC-mediated reactions in order to tune the functional properties of BSO. We also note that highly nonstoichiometric powders have reduced crystallinity, and contain $\mathrm{SnO}_{2}$ impurities resulting from the large tin excess (Figure $\mathrm{S} 6$ ).
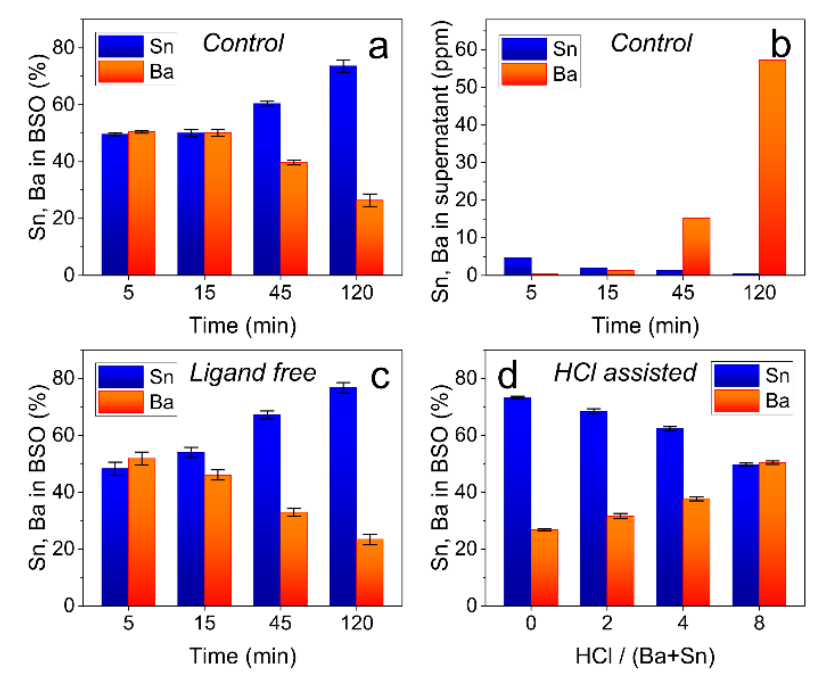

Figure 2. a) Tin and barium amount within BSO powders $(S n+B a=100 \%)$ as a function of mixing time prior to the ammonia addition. b) Sn and Ba amount within the supernatant of the reaction as a function of mixing time. c) Tin and barium amount within BSO powders as a function of mixing time for a reaction carried out without citric acid and (d) after 120 min of mixing time as a function of the amount of $\mathrm{HCl}$ used in the synthesis.

To completely understand of this phenomenon, we need to investigate what happens to the precursors over time. Tin (IV) chlorides when dissolved in water tend to form insoluble hydroxides through the release of hydrochloric acid. ${ }^{20}$ These hydroxide species are unstable and decompose into more stable $\mathrm{SnO}_{2} .{ }^{20}$ In our case this precipitation seems to start very quickly (within minutes), even though there is no visual change to the solution over several hours. We therefore postulate that some form of tin oxide/hydroxide cluster species is being formed in the aqueous hydrogen peroxide solution over time. When ammonia is added, this pre-formed tin species act as seeds for precipitation, culminating in BSO that contains excess tin. To test this hypothesis, we prepared a series of samples with increasing amount of hydrochloric acid, in order to limit or possibly prevent the formation of tin hydroxides before the addition of ammonia. As expected, for reactions conducted in the absence of $\mathrm{HCl}$ (after 2 hours of standing time, followed by addition of ammonia), the product showed a $\mathrm{Sn}$ :Ba ratio close to 3 , similar to what observed in previous reactions (Figure $2 a$ and 2c). Strikingly, the $\mathrm{Sn}$ :Ba ratio systematically decreases with the amount of $\mathrm{HCl}$ added, and eventually reaches $1: 1$. This corresponds to the correct stoichiometry and reflects the amount of precursors used in the synthesis (Figure $2 \mathrm{~d}$ ). While the solutions do not show evidence of precipitates before the addition of ammonia, the colloidal nature of the precursor solution prepared without $\mathrm{HCl}$ can be observed, confirming the formation of nano-sized clusters. A discussion on the nature of these precipitates is presented in the ESI as Figure S7. Attempts to mitigate this issue by changing the initial concentration of metal salts also proved ineffective, showing a large tin bias, and in one case, the occurrence of visible precipitates prior to ammonia injection (Figure S8 for details). However, pure stoichiometric samples were observed when adding $\mathrm{HCl}$ to the precursors. Acid stabilization also explains why reactions carried 
out with citric acid showed a slightly improved

temporal stability compared to reactions without any ligand, given the mildly acidic nature of citric acid. These results provide clear evidence that the prevention of tin-based clusters through an acid-mediated synthesis is critical to control the stoichiometry of BSO. Importantly, $\mathrm{HCl}$ has a positive effect on the morphology of BSO, albeit small, as shown in Figure S9. On average, slightly smaller and more homogeneous particles are obtained when using $\mathrm{HCl}$. This is especially evident when comparing BSO synthesized after long standing times: without extra $\mathrm{HCl}$, the particles are highly polydisperse, because of the large off-stoichiometric ratio and the presence of $\mathrm{SnO}_{2}$ impurities (Figure S6). Overall, we have demonstrated a strong tin bias in CSMC-mediated BSO syntheses, and related it to the precipitation of tin species over time. Hence, reproducible syntheses can be achieved through either the use of $\mathrm{HCl}$ and/or reducing the time between dissolution of precursors and addition of ammonia.

To further prove this aspect, we synthesized off-stoichiometric BSO with specifically defined $\mathrm{Sn}$ :Ba ratios using our optimized acid-mediated reaction. As evident from Figure $3 a$, we could tune the composition of BSO and achieve great agreement between the nominal and the measured composition, further highlighting the robustness of our protocol. The complete experimental and nominal compositions are reported in Table S2. All prepared materials are crystalline, both as CSMC precursors and as annealed powders (Figure $3 \mathrm{~b}$ and Figure S10). These results show that slight off-stoichiometry in BSO will not affect the crystallinity of either CSMC precursor or annealed $\mathrm{BSO}$, and therefore might not be detected unless compositional analysis is conducted. However, unintentional metal bias can lead to undesired functional properties, and therefore it is vital to adopt a synthetic protocol that enables the preparation of BSO with the desired chemical composition. Our optimized reaction is also easily scalable, enabling the preparation of several grams of material within a single batch, further improving the reliability of the method by reducing the intrinsic batch-to-batch variability typical of chemical syntheses.
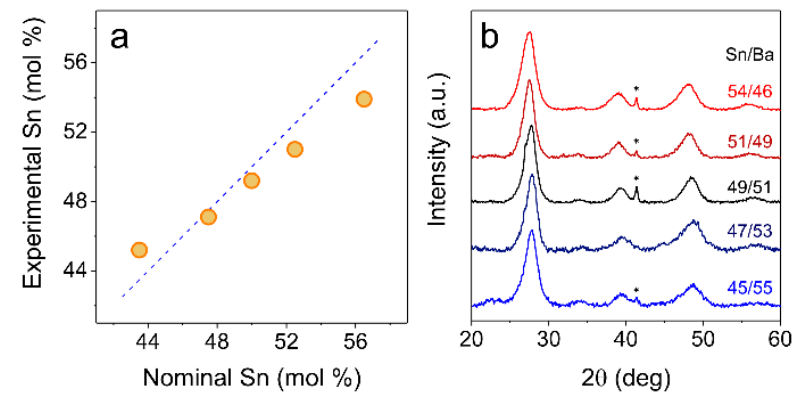

Figure 3. a) Nominal vs. experimental (from EDX) tin amount in molar \% (assuming Sn+Ba $=100 \%$ ) of BSO samples of various stoichiometries prepared with the optimised $\mathrm{HCl}$ recipe and with 1 hour standing time. The dashed line represents the identity line (nominal composition = experimental composition). b) Respective XRD patterns for offstoichiometric BSO. The reported values identify the experimental $\mathrm{Sn} /$ Ba ratio. The peak marked by a star is due to the XRD sample holder.

Having uncovered the key reaction parameters in the synthesis of BSO, we now extend this knowledge to the synthesis of La- doped nanoparticles. LBSO is one of the most promising emerging materials for transparent electrodes. It is therefore imperative to have reliable methods to synthesize LBSO with controlled doping levels. We synthesized LBSO and confirmed the synthesis of both the CSMC precursor and crystalline LBSO after annealing using XRD (Figure S11). We first analysed the efficacy of doping by synthesizing a series of LBSO samples with increasing amount of La precursor, keeping the total number of moles of metals ( $\mathrm{Ba}, \mathrm{Sn}, \mathrm{La}$ ) constant (see Experimental section). The results are presented in Figure 4a and $4 \mathrm{~b}$. All prepared materials show La doping levels very close to the expected value, highlighting the near stoichiometric conversion of lanthanum nitrate to lanthanum as a dopant within BSO (see also Table S3 for complete compositional details).

Although the conversion of the CSMC to BSO can be achieved at temperatures as low as $200^{\circ} \mathrm{C}, 10,15$ it is standard practice in the literature to anneal BSO precursors to $900{ }^{\circ} \mathrm{C}$ or above. In light of this, we annealed our LBSO precursors at $900^{\circ} \mathrm{C}$ and analyzed their optical properties using UV-Vis-NIR spectroscopy (Figure 4a). A prominent infrared (IR) absorption is observed in all doped samples, with its intensity increasing with La doping. This is also evident from the gradient analysis of the corresponding FTIR spectra (Figure $4 \mathrm{~b}$ and Figure S12). This IR absorption is due to free carriers provided by aliovalent doping, which give rise to a surface plasmon resonance (SPR). This SPR also imparts a distinctive green color to the powders, which is more intense at high La doping (Figure S12). This color has been previously observed in doped $\mathrm{BSO}, 13$ and in other plasmonic oxides such as indium tin oxide (ITO), doped $\mathrm{ZnO}$ and doped $\mathrm{SnO}_{2} \cdot{ }^{21,22,23,24}$ The presence of La dopants does not seem to significantly affect the size and shape of the nanoparticles, except for a slight increase in average size and polydispersity at high doping (Figure S13). The minor effect of La on the morphology of BSO, and the doping efficiency close to $100 \%$ are an indication of the small perturbation caused by La in the perovskite lattice.
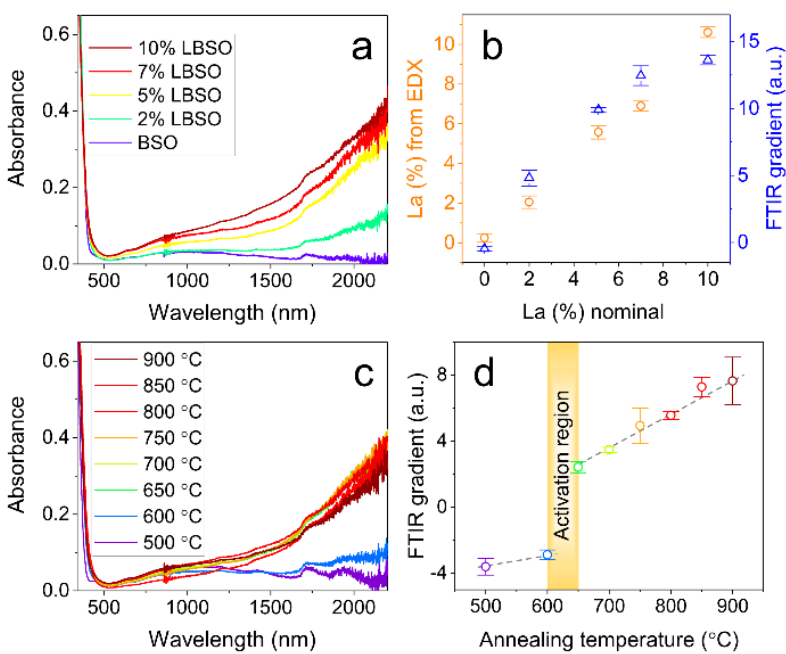

Figure 4. a) Absorption spectra and b) nominal vs real (from EDX) doping and gradient of FTIR spectra for LBSO powders annealed at $900{ }^{\circ} \mathrm{C}$ as a function of La amount. c) Absorption spectra and d) gradient of FTIR spectra for LBSO powders ( $10 \%$ doping) as a function of the annealing temperature (dashed lines are just a guide to the eye). 
Having confirmed the ability to efficiently doped BSO, we now analyze the effect of annealing. The conversion of the CSMC precursor into crystalline BSO is achieved between $200{ }^{\circ} \mathrm{C}$ and $300^{\circ} \mathrm{C}$. However, LBSO powders annealed at low temperatures, albeit crystalline, are white and do not display the IR absorption due to free carriers. This is an indication that a threshold temperature is required to activate La dopants within the BSO crystal structure. We therefore annealed the same LBSO CSMC precursor (10\% La doping) at temperatures up to $900{ }^{\circ} \mathrm{C}$. As shown in Figure $4 \mathrm{c}$ and $4 \mathrm{~d}$, the minimum required temperature to achieve dopant activation is $650{ }^{\circ} \mathrm{C}$. Powders annealed below $650{ }^{\circ} \mathrm{C}$ do not show any IR plasmonic features and are visually white. In contrast, samples annealed at $650{ }^{\circ} \mathrm{C}$ and above appear green and show an intense IR absorption, that is slightly more intense with increased annealing temperature (see also Figure S14). It is important to note that all these samples have been prepared from the same CSMC precursor (same batch), and that the amount of La in all samples is exactly the same as confirmed by EDX analysis (see Table S4 ESI).

In conclusion, through detailed analyses of the reaction conditions we have uncovered the critical role that acidmediated stabilization plays in achieving stoichiometrically controlled BSO nanoparticles. Our synthesis circumvents the reproducibility issues of currently available methods via the controlled speciation of the precursors. From this vantage point we have provided a reliable approach for the synthesis of BSO and La-doped BSO with tunable optical properties and highly controllable stoichiometry over all component atoms. This work provides the foundation for deeper studies into one of the most promising transparent conductive perovskite oxides.

EDG thanks RMIT University for a Vice Chancellor Fellowship. The Australian Research Council is acknowledged for financial support (DP190101864, DE170100164, DE150100427). The authors also acknowledge the use of the RMIT Microscopy and Microanalysis Facility (RMMF).

\section{Conflicts of interest}

There are no conflicts to declare.

\section{Notes and references}

1. S. Ismail-Beigi, F. J. Walker, S.-W. Cheong, K. M. Rabe and C. H. Ahn, APL Materials, 2015, 3, 062510.

2. H. J. Kim, U. Kim, H. M. Kim, T. H. Kim, H. S. Mun, B.-G. Jeon, K. T. Hong, W.-J. Lee, C. Ju, K. H. Kim and K. Char, Applied Physics Express, 2012, 5, 061102.

3. C. Park, U. Kim, C. J. Ju, J. S. Park, Y. M. Kim and K. Char, Applied Physics Letters, 2014, 105, 203503.

4. D. O. Scanlon, Physical Review B, 2013, 87, 161201.

5. A. Prakash, P. Xu, A. Faghaninia, S. Shukla, J. W. Ager lii, C. S. Lo and B. Jalan, Nature Communications, 2017, 8 , 15167.

6. S. Upadhyay, O. Parkash and D. J. J. o. M. S. L. Kumar, Journal of Materials Science Letters 1997, 16, 1330-1332.
7. M. G. Smith, J. B. Goodenough, A. Manthiram, R. D. Taylor, W. Peng and C. W. Kimball, Journal of Solid State Chemistry, 1992, 98, 181-186.

8. H. Mizoguchi, P. M. Woodward, C.-H. Park and D. A. Keszler, Journal of the American Chemical Society, 2004, 126, 9796-9800.

9. A. Kumar, B. P. Singh, R. N. P. Choudhary and A. K. Thakur, Materials Chemistry and Physics, 2006, 99, 150-159.

10. C. Huang, X. Wang, Q. Shi, X. Liu, Y. Zhang, F. Huang and T. Zhang, Inorganic Chemistry, 2015, 54, 4002-4010.

11. D. W. Kim, S. S. Shin, S. Lee, I. S. Cho, D. Kim, C. W. Lee, H. S. Jung and K. S. Hong, ChemSusChem, 2013, 6, 449-454.

12. L. Zhu, Z. Shao, J. Ye, X. Zhang, X. Pan and S. Dai, Chemical Communications, 2016, 52, 970-973.

13. L. Zhu, J. Ye, X. Zhang, H. Zheng, G. Liu, X. Pan and S. Dai, Journal of Materials Chemistry A, 2017, 5, 3675-3682.

14. S. S. Shin, J. S. Kim, J. H. Suk, K. D. Lee, D. W. Kim, J. H. Park, I. S. Cho, K. S. Hong and J. Y. Kim, ACS Nano, 2013, 7, 1027-1035.

15. S. S. Shin, E. J. Yeom, W. S. Yang, S. Hur, M. G. Kim, J. Im, J. Seo, J. H. Noh and S. I. Seok, Science, 2017, 356, 167-171.

16. Y. Zhang, H. Zhang, Y. Wang and W. F. Zhang, The Journal of Physical Chemistry C, 2008, 112, 8553-8557.

17. S. Sladkevich, V. Gutkin, O. Lev, E. A. Legurova, D. F. Khabibulin, M. A. Fedotov, V. Uvarov, T. A. Tripol'skaya and P. V. Prikhodchenko, Journal of Sol-Gel Science and Technology, 2009, 50, 229-240.

18. W. Lu and H. Schmidt, Journal of Sol-Gel Science and Technology, 2007, 42, 55-64.

19. Y. Ozaki, D. Kan and Y. Shimakawa, 2017, 121, 215304.

20. F. Séby, M. Potin-Gautier, E. Giffaut and O. F. X. Donard, Geochimica et Cosmochimica Acta, 2001, 65, 3041-3053.

21. J. Lee, S. Lee, G. Li, M. A. Petruska, D. C. Paine and S. Sun, Journal of the American Chemical Society, 2012, 134, 13410-13414.

22. E. Della Gaspera, A. S. R. Chesman, J. van Embden and J. J. Jasieniak, ACS Nano, 2014, 8, 9154-9163.

23. E. Della Gaspera, N. W. Duffy, J. van Embden, L. Waddington, L. Bourgeois, J. J. Jasieniak and A. S. R. Chesman, Chemical Communications, 2015, 51, 1236912372.

24. T. Nütz, U. z. Felde and M. Haase, The Journal of Chemical Physics, 1999, 110, 12142-12150. 